1 Fundação Oswaldo Cruz (Fiocruz), Escola Nacional de Saúde Pública Sergio Arouca (Ensp), Laboratório de Avaliação de Situações Endêmicas Regionais

(Laser) - Rio de Janeiro (RJ), Brasil.

anaclaudiafigueiro@ensp. fiocruz.br

2 Fundação Oswaldo Cruz (Fiocruz), Escola Nacional de Saúde Pública Sergio

Arouca (Ensp), Laboratório de Avaliação de Situações Endêmicas Regionais

(Laser) - Rio de Janeiro (RJ), Brasil.

mariasantosiris2@gmail.com

${ }^{3}$ Fundação Oswaldo Cruz (Fiocruz), Escola Nacional de Saúde Pública Sergio Arouca (Ensp), Laboratório de Avaliação de Situações Endêmicas Regionais

(Laser) - Rio de Janeiro (RJ), Brasil.

julianakabad@gmail.com

4 Fundação Oswaldo Cruz (Fiocruz), Escola Nacional de Saúde Pública Sergio Arouca (Ensp), Laboratório de Avaliação de Situações Endêmicas Regionais (Laser) - Rio de Janeiro (RJ), Brasil.

marlycruz12@gmail.com

5 Instituto de Higiene e Medicina Tropical (IHMT)

- Lisboa, Portugal.

zhartz@ihmt.unl.pt

\section{Avaliação da Rede Programa de Desenvolvimento e Inovação Tecnológica em Saúde Pública - Teias: inovação e produtos em questão}

\author{
Evaluation of Development Program and Technological Innovation in \\ Public Health Network - Teias: innovation and public health products
}

Ana Cláudia Figueiró1, Maria Aparecida dos Santos², Juliana Kabad³, Marly Marques da Cruz ${ }^{\mathbf{4}}$, Zulmira Hartz ${ }^{5}$

RESUMO O sistema de saúde requer inovações em processos e produtos para melhoria da gestão, da atenção e do estado de saúde da população. Nesse sentido, implementou-se a Rede Programa de Desenvolvimento e Inovação Tecnológica em Saúde Pública (PDTSP-Teias/ Fundação Oswaldo Cruz - Fiocruz), voltada para criação de produtos inovadores para o território de Manguinhos, Rio de Janeiro. A avaliação da rede objetivou mapear os produtos dos projetos como Inovação em Saúde Pública. Considerou-se que os conceitos 'Inovação' e 'Produtos de saúde pública' estavam insuficientemente definidos nos documentos e entendimento de gestores e pesquisadores. Esse fato se mostrou relevante para a não entrega dos produtos acordados, sendo as publicações científicas entendidas como principal produto de pesquisa.

PALAVRAS-CHAVE Avaliação da pesquisa em saúde. Inovação. Rede social. Síntese de produtos.

ABSTRACT Health system requires proposals for innovations in processes and products to improve management, care and population's health status. In this sense the Development and Technological Innovation Program in Public Health Network (PDTSP-Teias/Oswaldo Cruz Foundation - Fiocruz) was implemented as to create innovative products for Manguinhos's territory, Rio de Janeiro, Brazil. The evaluation of the Network aimed at mapping the products of the projects such as Innovation in Public Health. The concepts of 'Innovation' and 'Public health products' were considered poorly defined both in the documents and in the understanding of managers and researchers. This fact revealed to be relevant to the non-delivery of the agreed products. Scientific publications were considered sufficient as research product.

KEYWORDS Health research evaluation. Innovation. Social networking. Product synthesis. 


\section{Introdução}

O fortalecimento do Sistema Único de Saúde do Brasil (SUS) requer das instituições de pesquisa, sobretudo daquelas apoiadas por fomentos públicos, a proposição de ideias inovadoras em processos e produtos tanto para a melhoria na qualidade das práticas de gestão e atenção no SUS como do estado de saúde da população brasileira. Com esse propósito, foi desenvolvido o Programa de Desenvolvimento e Inovação Tecnológica em Saúde Pública - Teias (PDTSP-Teias) durante o período de 2010 a 2012, visando a estabelecer uma rede de projetos para elaboração de produtos no âmbito da Atenção Básica de Saúde, para o território de Manguinhos, Rio de Janeiro, que pudesse ser expandida para outras regiões do município (SANTOS; GOLDSTEIN; RABELLO, 2016).

Ao fim do programa, realizou-se uma pesquisa avaliativa com o objetivo de compreender os processos e resultados do modo de gestão implementado pela Rede PDTSPTeias e os produtos gerados pelas pesquisas para a inovação tecnológica pretendida em saúde. Este artigo apresenta a primeira parte dos achados da pesquisa avaliativa, que se refere ao entendimento dos envolvidos no programa, pesquisadores e gestores quanto aos conceitos de inovação e produtos em saúde pública para o SUS, sejam eles inovadores ou não, e apresenta possibilidades de reflexão utilizáveis para outras equipes de pesquisa em saúde.

O Programa de Desenvolvimento e Inovação Tecnológica em Saúde Pública (PDTSP), vinculado à Vice-Presidência de Pesquisa e Laboratórios de Referência da Fundação Oswaldo Cruz (VPPLR/Fiocruz), foi concebido, conforme Santos, Goldstein e Rabello (2016), como uma modalidade de apoio à pesquisa orientada à produção de conhecimento e inovações capazes de fazer frente às situações vistas como promotoras das iniquidades de saúde. Implantado em 2002, o Programa atuou com seis projetos até o ano de 2008, reformulou-se e adotou um novo modelo de gestão entre 2009 e 2010. No âmbito do Programa Território Escola Manguinhos (Teias), atualizou-se como Rede PDTSP-Teias.

Dessa maneira, a Rede PDTSP-Teias surgiu no bojo de uma política pública lançada pelo governo federal, em 2009, intitulada Redes Regionalizadas e Territórios Integrados de Atenção à Saúde - Teias. No Rio de Janeiro, essa política foi implantada por parceria entre o município e a Fiocruz, por meio da Escola Nacional de Saúde Pública Sergio Arouca (Ensp). A atuação do Teias visou à inserção da noção de territorialização tanto no entendimento das condições de vida de determinada população como nas estratégias de intervenção em saúde pública. Com base nessa política, a Rede PDTSPTeias propôs-se como uma experiência de gestão da produção do conhecimento e utilização dos seus produtos, métodos e práticas na intenção do aprimoramento do modelo do Programa Teias Escola Manguinhos.

Devido ao caráter original da proposta de gestão de projetos de pesquisa em rede e à expectativa de produtos de pesquisa a serem aplicados para a saúde pública, demandou-se a realização da pesquisa avaliativa. Nessa disposição, um dos desafios da avaliação era o de encontrar fios que pudessem conduzir explicações sobre as dificuldades de alcançar um dos resultados pretendidos, qual seja, a entrega dos produtos para o SUS.

A pesquisa avaliativa seguiu por dois movimentos de análise: redes e inovação. $\mathrm{Na}$ primeira parte, apresentada em outro trabalho (FIGUEIRÓ ET AL., 2016A), o objetivo foi o de compreender por quais caminhos, relações, interesses e controvérsias desenvolveu-se a gestão da Rede PDTSP-Teias como rede sociotécnica e os seus resultados (LATOUR, 2005; FIGUEIRÓ ET AL., 2016B). Neste artigo, apresenta-se o segundo movimento, o actante Inovação e sua expressão nos projetos como produtos de saúde pública para o território de Manguinhos. 
A opção pela Teoria do Ator Rede (TAR) como abordagem teórica e metodológica justifica-se pela identificação do objeto de estudo como uma 'rede sociotécnica', caracterizada pela maneira como elementos heterogêneos se reúnem e se esgarçam, para, em outro momento, adquirir novas formas, por vezes inovadoras (LATOUR, 2005; POTVIN ET AL., 2005). Assim, quando se iniciou a pesquisa avaliativa, a Rede PDTSP-Teias encontrava-se em um momento de esgarçamento, de baixa confiança nas relações e importantes controvérsias. Dentre essas controvérsias sobressaía o entendimento do que viria a ser um produto inovador em saúde pública e a consequente dificuldade para a entrega desse produto, o que exigiu um aprofundamento do tema, constituindo-se em um actante da rede.

A TAR permitiu, assim, observar as entidades e materialidades como relacionais e explorar a configuração e reconfiguração dessas relações (POTVIN; CLAVIER 2012), reconhecendo as diferenças e dificuldades que envolveram os dois mundos: o da gestão e o da produção do conhecimento científico.

Para esta pesquisa, a proposta foi a de tatear um terreno híbrido "repleto de forças geradoras e relacionais, forças em tensão um mundo em rede" (LAW; 2004, P. 10), composto pela presença de 'actantes' humanos, os atores sociais, e não-humanos - um conhecimento, um novo financiamento, equipamentos, documentos etc. Um actante é definido quando deixa traços, rastros na rede, e, assim, pode ser seguido. Os actantes produzem efeitos, modificam e são modificados pela rede e são esses elementos que devem fazer parte de uma descrição que propõe trabalhar com a TAR (LATOUR, 2001).

O conceito de Inovação, tomado como um actante neste estudo, compreende a introdução e a exploração de novos produtos, processos, insumos e formas de organização. Desse modo, exige implementação e impactará os custos, serviços, processos de atendimento etc. Quando dizem respeito ao campo da saúde pública, produtos e processos são, por vezes, tangíveis e, em tantas outras, são intangíveis, imateriais e abstratos. Produtos classificados como tangíveis podem ser vistos ou manuseados pelo potencial interessado: um filtro diferenciado, uma vacina, um aparelho etc. (BRASIL, 2001). Produtos intangíveis, tais como uma nova rotina ou processo de trabalho, possuem menor reconhecimento e retorno dentro das instituições de fomento à pesquisa, sendo, por vezes, desconsiderados.

Dessa maneira, no desafio de encontrar a rede sociotécnica constituída no programa avaliado e seus actantes, a TAR serviu como ferramenta metodológica para compreender como os produtos inovadores em saúde pública esperados pelos projetos de pesquisa foram alcançados ou não. A produção científica em rede - de projetos e grupos de pesquisa - para geração de produtos inovadores e sua aplicação pode ser representada por meio das translações do conhecimento. Neste estudo, esse conceito refere-se a

um processo dinâmico e interativo que envolve a síntese, a disseminação, a partilha de conhecimentos e a aplicação dos conhecimentos de acordo com a ética e o intuito de melhorar a saúde da população, oferecer meIhores produtos e serviços de saúde e reforçar o sistema de saúde. (CIHR, 2012).

Nesse sentido, os conceitos de inovação e de produtos em saúde pública serão problematizados como actantes da rede sociotécnica, considerando a centralidade para o desenvolvimento e resultados esperados pela Rede PDTSP-Teias.

\section{Métodos}

Para a realização deste estudo, foi utilizada a abordagem qualitativa (SCHWANDT, 2006), em uma perspectiva participativa e formativa (THIOLLENT, 1987) tanto pela natureza do objeto em questão como pela intenção de 
envolvimento dos envolvidos e potenciais usuários da avaliação. O referencial teórico e metodológico da Teoria do Ator Rede (TAR) (LATOUR, 2005) foi central por possibilitar a pesquisa de um objeto em ação, que se dá em rede, na conexão entre conhecimento e prática.

Optou-se pela participação contínua dos coordenadores e pesquisadores envolvidos na Rede ao longo da realização do estudo, considerando que a pesquisa participativa tem sido indicada como abordagem relevante para avaliar intervenções com características inovadoras (MANTOURA; POTVIN 2013). Para isso, empreendeu-se a realização de quatro oficinas sobre 'Produtos em Saúde Pública' durante o processo da pesquisa. Nessa ocasião, promoveu-se o compartilhamento e produção reflexiva entre os participantes (BOURDIEU; WACQUANT 1992) sobre os achados intermediários da avaliação e possíveis aplicações no desenvolvimento dos projetos.

Para a coleta de dados, foram utilizadas as técnicas de análise documental, entrevista semiestruturada com os dois coordenadores do programa e instrumento de pesquisa (CIHR, 2012) para discussão e resposta pelas equipes de pesquisa no decorrer das oficinas do projeto. Dos 14 projetos que constituíram a Rede PDTSP, oito grupos de pesquisa participaram das oficinas e da coleta de dados.

Para a análise documental, foi realizado o levantamento bibliográfico sobre o tema inovação e de material de interesse para o estudo, como textos produzidos pela coordenação do programa e documentos oficiais e não oficiais - atas e relatos de reunião, relatórios de gestão e material de divulgação, legislações, documentos institucionais da Fiocruz e ministérios.
O instrumento utilizado para coleta de dados junto aos grupos de pesquisa contemplou as seguintes questões: 'identificação' dos projetos e dos produtos; 'quem': Participantes e público que se pretende alcançar com o projeto; 'por que': Objetivo pretendido com a troca de conhecimentos; 'como': Escolha do formato e dos meios; 'oportunidades' e 'barreiras': O que pode favorecer e limitar o compartilhamento do conhecimento; 'impacto': Alcance do compartilhamento dos conhecimentos do projeto.

O roteiro de análise documental e entrevista com os coordenadores incluiu os seguintes aspectos: trajetória dos envolvidos; escopo teórico e conceitual; objetivos e resultados esperados; critérios de seleção e avaliação dos projetos; cronologia dos eventos da Rede e dos projetos: reuniões, eventos, atividades; fluxo de informações; organização dos trabalhos individuais e coletivos: grupos de trabalho, grupos de pesquisa, outras redes; protocolos estabelecidos: formulários, relatórios, prazos etc.

O conteúdo dos dados coletados foi analisado via leituras sucessivas, procurando olhar para os temas previstos nas categorias de análise evidenciadas no quadro 1. Identificou padrões emergentes, num processo de codificação aberta, bem como aprofundou as articulações estabelecidas entre os dados e os referenciais teóricos da pesquisa (BARDIN, 2011). O estudo, comprometido com uma pesquisa participativa e formativa, compartilhou os resultados parciais com os interessados ao longo da sua realização, passando a desenvolver-se também como projeto de apoio à gestão. 
Quadro 1. Ficha conceitual das categorias de análise

INTERESSES DOS ATORES Essa categoria expressa as lógicas do comportamento dos atores, cujas estratégias

O QUE ESTÁ EM JOGO dependem de seus próprios interesses e recursos, assim como das estratégias e táticas adotadas por outros atores e do contexto.

A lógica de ação é uma lógica de interesse: 0 ator maximiza seus ganhos e minimiza suas perdas, ao preço do conflito, da negociação (POTVIN, 2005, 2012; LAW, 2004; LATOUR, 2005).

INTERAÇÕES

AÇÕES

INSCRIÇÕES
A emergência de uma rede requer que os atores façam alianças, conexões com outras redes, produzam fatos, inscrições ao visando o êxito do projeto. A categoria 'Interações' revela as relações, conexões estabelecidas entre atores, instituições e estabilização do fato em análise, incluindo a expansão da rede na medida da exploração dos mundos possíveis (LATOUR, 2005; LAW, 2004)

Identifica as práticas, atividades, processos de trabalho realizados pelos atores na emergência, implementação, estabilização de um evento.

Entende-se inscrição como todos os tipos de transformação que materializam uma entidade num signo, num arquivo, num documento, num pedaço de papel, num traço. Usualmente, mas nem sempre, as inscrições são bidimensionais, sujeitas à sobreposição e combinação. São sempre móveis, isto é, permitem novas translações e articulações, ao mesmo tempo em que mantêm intactas algumas formas de relação (LATOUR, 2001, 2005).

CONSEQUENNCIAS - $\quad$ As consequências relatam as mudanças no programa ou intervenção decorrente da EFEITOS

Fonte: Adaptado de Figueiró et al. (2016b).

\section{Resultados e discussão}

Um primeiro achado a ser destacado foi a necessidade de ampliação da compreensão sobre o conceito de 'Inovação' no campo da saúde pública, por ser o eixo da implementação do PDTSP desde a sua origem em 2001. Uma vez que produtos inovadores eram o resultado esperado dos projetos de pesquisa que concorreram à chamada para conformação da Rede PDTSP-Teias, o conceito de inovação constituiu-se como um fio condutor para adentrar a rede sociotécnica. Mais adiante, esse mesmo fio identificaria um nó: a dúvida sobre o que viria a ser um produto em saúde pública para o SUS.

Embora o termo 'Inovação' estivesse presente em muitos registros e inscrições do PDTSP - editais, outdoors, sites, títulos de trabalhos -, sua compreensão não parecia tão fundamental para os gestores nem para os pesquisadores da rede. Os coordenadores do programa não tinham claro uma definição sobre que inovação estava se esperando dos projetos da rede nem de seu conceito na saúde pública. Essa constatação revelou a importância da construção do conceito por meio de uma linha do tempo, que expressou suas transformações, controvérsias e adequações; a inovação saiu do parque industrial para a área da saúde, até sua entrada na Fiocruz e, em particular, nessa rede de projetos para o território de Manguinhos.

\section{O conceito de inovação e sua adoção institucional}

A linha do tempo construída com base na análise documental, teve como objetivo descrever a origem e o estabelecimento do conceito de inovação no Ministério da Ciência e Tecnologia até sua entrada no Ministério da Saúde, bem como sua presença nos documentos da Fiocruz (figura 1). 
Figura 1. Linha do tempo do conceito inovação na Fiocruz

\section{Linha do Tempo - Inovação}

\begin{tabular}{|c|c|}
\hline 1992 & $\begin{array}{l}\text { Manual de Oslo 1a edição. } \\
\text { Inovação tecnológica de produto e processo (TPP) } \\
\text { na indústria de transformação }\end{array}$ \\
\hline 1997 & $\begin{array}{l}\text { Manual de Oslo 2a edição. } \\
\text { Expandiu para o setor de serviços }\end{array}$ \\
\hline
\end{tabular}

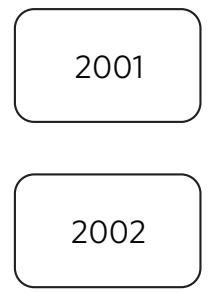

PDTSP - Programa de Desenvolvimento Tecnológico em Saúde Pública

Política Nacional de Ciência, Tecnologia e Inovação em Saúde

Lei da Inovação

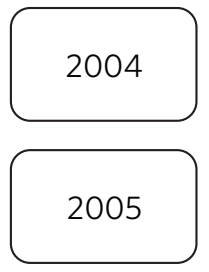

Il Conferência Nacional de Ciência, Tecnologia e Inovação em Saúde

3a edição do Manual de Oslo.

Expandiu para inovação do marketing e inovação organizacional

Plano Quadrienal 2005 - 2008

Fiocruz

Fiocruz Manual para Pesquisa Pintec, IBGE

2008

2009
Projeto Inovação e Desenvolvimento Tecnológico para a Gestão de Territórios integrados de Atenção à Saúde (PDTSP-Teias)
Inovação: uma ideia, uma prática ou um artefato material percebido como novo, relevante e único adotado em um determinado processo, área ou por toda a organização

Transformação de ideias em produtos, processos e abordagens tecnologicamente novos ou significativamente aprimorados, visando soluções para os problemas no atendimento às necessidades de saúde da população brasileira

Missão do PDTSP: Apoiar a inovação tecnológica no campo da saúde pública, visando soluções para as necessidades de saúde da população brasileira

Inovação: introdução de novidade ou aperfeiçoamento no ambiente produtivo ou social que resulte em novos produtos, processos ou serviços, com base no art. 2으, IV, Lei ํำ10.973/04.

Inovação: introdução no mercado de um produto (bem ou serviço) novo ou substancialmente aprimorado ou pela introdução na empresa de um processo novo ou substancialmente aprimorado.

Inovação introdução de novidade ou aperfeiçoamento no ambiente produtivo ou social que resulte em novos produtos, processos ou serviços

Inovação: introdução no mercado de um produto (bem ou serviço) novo ou substancialmente aprimorado ou pela introdução na empresa de um processo novo ou substancialmente aprimorado

Transformação de ideias em produtos, processos e abordagens tecnologicamente novos ou significativamente aprimorados, visando soluções para os problemas no atendimento às necessidades de saúde da população brasileira

Principal meta do PDTSP-Teias: contribuir para a formação de atores críticos, comprometidos com a mudança e com a inovação, capazes de requalificar suas práticas e reinventá-las cotidianamente, com consequente produção de conhecimento 
Ao seguir o rastro pela linha do tempo, observa-se que o tema inovação consolidou-se no Manual de Oslo, desenvolvido conjuntamente pelo Eurostat e pela Organization for Economic Cooperation and Development (OECD). A produção sobre o tema compõe uma série de três manuais dedicados à mensuração e interpretação de dados relacionados à ciência, tecnologia e inovação (1992, 1997 e 2005), nos quais os Ministérios da Ciência e Tecnologia de Saúde no Brasil se inspiram para incluir a temática em suas políticas de fomento.

O Ministério da Saúde fortaleceu a ideia da necessidade de inovação no campo da saúde, contemplando o tema no documento Política Nacional de Desenvolvimento e Tecnologia em Saúde, no ano de 2002. No mesmo ano, a Fiocruz lançou o Programa de Desenvolvimento Tecnológico em Saúde Pública (PDTSP) e indicou como missão

Apoiar a inovação tecnológica no campo da saúde pública, definida como a transformação de ideias em produtos, processos e abordagens tecnologicamente novos ou significativamente aprimorados, visando soluções para as necessidades de saúde da população brasileira. (FIOCRUZ, 2002).

Por sua vez, a formulação da Lei da Inovação $\mathrm{n}^{\mathrm{o}} 10.973$, de 2 de dezembro de 2004, apresentou um conjunto de medidas de incentivos à inovação científica e tecnológica, com esforço concentrado em Pesquisa, Desenvolvimento e Inovação. Ainda no mesmo ano, aconteceu a II Conferência Nacional de Ciência, Tecnologia e Inovação em Saúde, que apresentou uma agenda de prioridades de pesquisa e se propôs a instrumentalizar os atores do campo da saúde. No mesmo período, o Plano Quadrienal 20052008 da Fiocruz mostrou forte indicativo de que a ideia de inovação de produtos e processos deveria estar presente nas pesquisas em saúde pública. Essa proposta se acentua em 2009, quando a instituição outorgou o Projeto de Inovação e Desenvolvimento Tecnológico para a gestão de territórios integrados de Atenção à Saúde, designado PDTSP-Teias.

A chamada para projetos da rede PDTSPTeias de 2010-2012 marcou a ideia de produto inovador, definindo inovação como

transformação de ideias em produtos, processos e abordagens tecnologicamente novos ou significativamente aprimorados, visando soluções para os problemas no atendimento às necessidades de saúde da população brasileira. (SANTOS; GOLDSTEIN; RABELLO, 2016, P. 312).

Esperava-se, então, a criação e implantação de produtos inovadores em saúde pública para o SUS no território de Manguinhos, porém não estava bem explicitado o que seria inovação, bem como o que viria a ser um produto para o SUS no âmbito do território. Dessa maneira, pode-se notar que a definição de Inovação, utilizada pelo PDTSP-Teias e aplicada à chamada de convocação dos projetos, teve por alicerce $o$ conceito de Inovação Tecnológica existente no documento do PDTSP de 2002. Outras ponderações sobre inovação em saúde pública foram buriladas quando do Relatório Final do VI Congresso Interno da Fiocruz, de outubro de 2010.

As inovações de produtos no setor de serviços podem incluir melhoramentos importantes no que diz respeito a como elas são oferecidas - por exemplo, em termos de eficiência ou de velocidade; à adição de novas funções ou características em serviços existentes; ou à introdução de serviços inteiramente novos (BRASIL, 2001). Outro conceito, e mais próximo do entendimento sobre o ocorrido na Rede PDTSP-Teias, é o de 'atividade inovativa', que predominou ao de inovação de produtos ou de processos propriamente dito. Aa atividades inovativas compreendem todos os passos científicos, tecnológicos, organizacionais, financeiros e comerciais, inclusive o investimento em novos conhecimentos, que, efetiva ou potencialmente, levem à introdução de produtos ou processos 
tecnologicamente novos ou substancialmente melhorados. As atividades inovativas mais destacadas são: aquisição e geração de novos conhecimentos relevantes para a instituição; preparações para a produção; marketing dos produtos novos ou melhorados (OECD, 2005).

Por outro lado, considera-se que uma inovação tecnológica de produto ou processo foi realizada se tiver sido introduzida no campo (inovação de produto) ou utilizada no processo da produção (inovação de processo) e não se afixar na atividade inovativa (OECD, 2005). No caso da Rede PDTSP-Teias, as pesquisas, à exceção de duas, não devolveram produtos para o território Manguinhos. No entanto, existiu uma intensa produção de conhecimento científico sobre o campo e a gestão participativa em rede, o que pode ser considerado como atividade inovativa, propícia para gerar produtos e processos inovadores.

Desse modo, as discussões sobre o que viria a ser produtos para o SUS e em que medida seriam inovadores foram aprofundadas nas quatro oficinas decorrentes dos primeiros resultados da avaliação. Nota-se, contudo, que a falta do conhecimento tácito sobre o que viria a ser 'inovação', identificada na pesquisa avaliativa da Rede do PDTSP-Teias, pode transformar a procura pelo conceito em um 'actante' que conduziu gestores e pesquisadores ao reconhecimento e reconfiguração do que viria a ser um produto inovador para o SUS.

\section{Concepções e descobertas sobre produtos para o SUS}

Percorrendo o fio do actante Inovação na rede sociotécnica, outro desafio foi explicitar os produtos esperados como resultantes dos projetos. Constituiu-se um exercício para pesquisadores e gestores compreender as especificidades de produtos em saúde pública, sendo necessária a assinatura de uma carta de intenção para a entrega dos produtos acordados. A partir do processo da pesquisa avaliativa, quando do compartilhamento dos achados parciais e das reflexões nas oficinas, os produtos puderam ser explicitados e tornaram-se visíveis. Nesse processo, a constatação acerca dos produtos tangíveis e intangíveis foi um exercício realizado conjuntamente entre avaliadores, coordenadores do programa e pesquisadores.

Dentre as descrições e conceitos de inovação existentes, $\mathrm{o}$ interesse mais frequente recai sobre os produtos tangíveis - vacinas, medicamentos, insumos. Produtos inovadores intangíveis, i.e. processos (BRASIL, 2008), são de mais difícil mensuração e visibilidade, não permitindo demonstrar para o Estado e instituições, inclusive a Fiocruz, o cálculo da economia que fornecem ao País ou sua importância para o desenvolvimento da saúde pública. Ressaltase, ainda, que, entre a produção de inovações tangíveis e os potenciais usuários, muitas iniciativas e mudanças são necessárias. Para que novos produtos sejam assimilados e alcancem os efeitos esperados na solução de problemas e necessidades da população, inúmeros processos inovadores fizeram as pontes, as ligações entre as fronteiras da sociedade, governo, indústria, pesquisadores, amostragens, consolidação e publicações.

Para a maior parte dos pesquisadores o resultado dos projetos, ou produtos esperados, estava associado a um fazer científico, traduzido principalmente em publicações científicas e numa variedade de materiais que sintetizam o processo de trabalho das pesquisas - (artigos, capítulos de livro, apresentação em congressos). Tal realidade por ser confirmada nas respostas abaixo sobre produtos tangíveis e intangíveis, em que os pesquisadores consideraram como:

Tangíveis: publicações, método, mapas. Intangiveis: conhecimentos sobre a realidade local pelos próprios residentes. (Grupo A-Oficina 1).

Tangivel: artigos, manual de rotina para o serviço de saúde, material didático para a população. Intangível: a proposta de cuidado nutricional. (Grupo B-Oficina 1). 
A discussão acerca desse tópico propiciou um debate sobre quanto um produto é tangível, ou seja, um resultado material - por exemplo, material didático a ser utilizado nas escolas - ou intangível, como no caso de estratégias de empoderamento da população sobre as informações em saúde para que fossem fortalecidas suas relações com diferentes esferas do poder público.

De forma consensual, o artigo científico é citado como exemplo de produto produzido para o campo, embora não se consiga explicitar seu público de interesse, apesar de se saber que um artigo científico é produzido e divulgado para ser compartilhado pela comunidade científica. Quanto aos exemplos de produtos e seus propósitos, surgiram referências a diferentes tipos de materiais em todos os discursos, que se mostraram variáveis conforme o público a ser direcionado.

Um aspecto diferencial nas respostas foi a importância de produtos acessíveis à compreensão da população de uma maneira geral, incluindo gestores, trabalhadores e moradores do território, a partir da noção que as evidências científicas precisam ser traduzidas de modo a serem incorporadas aos processos decisórios. Essa reflexão direcionou o tema da translação do conhecimento tal como definido anteriormente. No exemplo abaixo, reproduzem-se as diferentes formas de apresentação dos produtos em um dos projetos participantes das oficinas:

- Produto original: Contribuições para um diagnóstico socioambiental em Manguinhos; Resultado e registro das estórias de campo e adaptação metodológica; Mapas com informações ambientais.

- Termo de Compromisso: Apresentará até 14 de dezembro de 2012 um relatório final da pesquisa e em fevereiro de 2013 os produtos: Uma sistematização do processo de trabalho para a construção do diagnóstico sócio ambiental; Texto para integrar a publicação final da Rede PDTSP-Teias.
- Produto elaborado após a 1a Oficina sobre Inovação e Produto em Saúde Pública: Diagnóstico da situação social e ambiental. Mapas temáticos sobre os diversos temas específicos do projeto. Publicações/material didático de conteúdo acessível à população local e aos trabalhadores da saúde no território.

Nesse sentido, as oficinas indicaram o que viria a ser um produto para o SUS e ajudaram a pensar tanto nos produtos como na inclusão dos interessados nos projetos. Aprofundouse a ideia de participação dos interessados - gestores, profissionais, usuários - desde a concepção dos projetos, movimento que parecia ser difícil de experimentar em um fazer acadêmico convencional.

Cabe ressaltar que dois projetos apresentavam características inovadoras, dando importância a um modelo de pesquisa intervencional participativa (MONTOURA; POTVIN, 2013), e uma delas envolveu a comunidade em todo o seu processo.

Por fim, um ponto de destaque levantado pelos pesquisadores e coordenadores, diz respeito à necessidade de investimentos institucionais para que as pesquisas resultem em produtos e em inovação para o campo da saúde pública. A translação de conhecimento entre os diferentes mundos e atores requer aprendizado e experimentação. Nesse sentido, não bastam iniciativas de pesquisa individuais e focadas se a instituição não tomar para si a responsabilidade de realizar a articulação entre atividade e produção técnico-científica, gestão dos serviços públicos e demandas da população do território.

\section{Conclusões e recomendações}

Ao final do percurso avaliativo, identificou-se que a própria gestão da Rede PDTSP-Teias se mostrou uma importante atividade inovativa em saúde pública. Reconhecendo-se que 
não houve efetivamente implementação dos produtos dos projetos no território, pode-se concluir que a elaboração de 'produto inovador' como definido inicialmente não foi alcançado. Porém, alguns projetos avançaram para essa possibilidade, principalmente aqueles desenvolvidos de forma mais participativa, tendo sido recomendado o apoio institucional para implementação de alguns dos produtos em uma etapa seguinte do programa.

Ao levar em consideração os conceitos aqui apresentados identifica-se a circulação do conhecimento na produção e publicação científica a partir dos projetos, etapa necessária à translação. Espera-se que os conhecimentos circulantes alcancem outras audiências e contribuam para melhores práticas em saúde pública.

Apesar das discussões sobre 'Produtos em saúde pública' ao longo das oficinas do projeto, ainda assim restaram dúvidas para os pesquisadores e gestores a respeito do que seriam produtos para o SUS e em que medida seriam inovadores. A ligação entre academia-serviço-gestão-comunidade ainda é pouco clara, e tal dificuldade pode ser devida à não valorização de publicação científica como produtos de pesquisa em saúde pública ou, ainda, de produtos ou processos inovadores tangíveis, como insumos, produção de medicamentos, vacinas, equipamentos etc.

O Ministério da Ciência, Tecnologia e
Inovação, a Fiocruz e as agências financiadoras podem colaborar para a mudança de visão e ação de pesquisadores, valorizando os processos menores, mais baratos e intangíveis. Por parte dos pesquisadores, identifica-se a necessidade de experimentar métodos de pesquisa que favoreçam a participação ativa dos interessados, desde que o projeto tenha por objetivo produtos para saúde pública. Essa participação pode se dar desde a definição do problema e pergunta do estudo, o objeto e objetivos da pesquisa, abordagem metodológica e validação dos resultados, de maneira a potencializar um produto final que seja útil e utilizável no território e traga melhoria para a população. Compreende-se que os métodos, suas regras e práticas produzem uma realidade que se reverte em objetos passíveis de pesquisa; assim, "os métodos, não apenas descrevem, mas também ajudam a produzir a realidade que a eles entendem" (LAW, 2004, P. 5).

A rede de pesquisa de produtos para o território de Manguinhos se movimenta, desterritoraliza e reterritorializa (DELEUzE; GUATTARI, 1997), muda de cara, mas continua. Hoje está se realizando em uma nova rede. Esta, desde o seu edital, preconiza que o produto se divulgue além das publicações científicas, observando a inteligibilidade dos conceitos e induzindo os pesquisadores a pensarem e incluírem o território não como objeto distante e sim coparticipante. 


\section{Referências}

BARDIN, L. Análise de Conteúdo. São Paulo: Edições 70, 2011.

BOURDIEU, P.; WACQUANT, L. An invitation to reflexive sociology. Chicago: University of Chicago Press, 1992.

BRANDÃO, C. Pesquisa Participante. São Paulo: Brasiliense, 1985.

BRASIL. Ministério da Ciência, Tecnologia e Inovação. Ciência, tecnologia e inovação: desafio para a sociedade brasileira: livro verde. Brasília, DF: Ministério da Ciência e Tecnologia; Academia Brasileira de Ciências, 2001.

Ministério da Saúde. Secretaria-Executiva. Glossário temático: Sistema de Planejamento, Monitoramento e Avaliação das Ações em Saúde (Sisplam). Brasília, DF: MS, 2008. (Série A. Normas e Manuais Técnicos)

CANADIAN INSTITUTES OF HEALTH RESEARCH (CIHR). Guide to Knowledge Translation Planning at Integrated and End-of-Grant Approaches. 2012. Disponível em: <www.irsc-cihr.gc.ca $>$. Acesso em: 20 dez. 2016.

CLAVIER, C.; SÉNÉCHAL, Y.; POTVIN, L. A theorybased model of translation practices in public health participatory research. Sociol. Health Illn, Henley-onThames, v. 34, n. 5, p. 791-805, 2011.

DELEUZE, G.; GUATTARI, F. Mil Platôs. Capitalismo e Esquizofrenia. Rio de Janeiro: Editora 34, 1997.

FIGUEIRÓ, A. C. et al. Avaliação da Rede PDTSPTeias: contribuição ao debate sobre construção do conhecimento e de produtos para o SUS. In: SANTOS, I. S.; GOLDSTEIN, R. A. (Org.). Rede de Pesquisas em Manguinhos: sociedade, gestores e pesquisadores em conexão com o SUS. São Paulo: Hucitec, 2016a. p. 293-322.

FIGUEIRÓ, A. C.; OLIVEIRA, S. R. A.; HARTZ,
Z. A tool for exploring the dynamics of innovative interventions for public health: the critical event card. Int J Public Health, Basileia, v. 62, n. 2, p. 177-186, ago. 2016. Disponível em: <http://link.springer.com/ article/10.1007/s00038-016-0861-5>. Acesso em: 15 fev. 2017.

\section{FUNDAÇÃO OSWALDO CRUZ (FIOCRUZ). PDTSP:} Programa de Desenvolvimento Tecnológico em Saúde Pública, 2002. Disponível em: <http://www.castelo. fiocruz.br/vpplr/pdtsp.php>. Acesso em: 10 fev. 2017.

LAW, J. After Method: Mess in Social Science Research. New York: Taylor \& Francis, 2004.

Making a Mess with Method. Bailrigg: Lancaster University, 2003.

LATOUR, B. Reassembling the social: an introduction to Actor-Network-Theory. London: Oxford University Press, 2005

A esperança de Pandora: ensaios sobre a realidade dos estudos científicos. Bauru: Edusc, 2001.

ORGANIZATION FOR ECONOMIC COOPERATION AND DEVELOPMENT (OECD). Annual report 2005. Paris: OECD, 2005. Disponível em: <https://www.oecd. org/about/34711139.pdf>. Acesso em: 15 fev. 2017.

MANTOURA, P.; POTVIN, L. A realist-constructionist perspective on participatory research in health promotion. Health Promot Int, Oxford, v. 28, n. 1, p. 61-72, 2013.

POTVIN, L.; CLAVIER, C. La théorie de l'acteurréseau. In: AUBRY, F.; POTVIN, L. (Ed.). Construire l'espace socio-sanitaire: expériences de recherche dans la production locale de la santé. Montréal: Presse de l’Université de Montréal, 2012. p. 77-98.

POTVIN, L. et al. Integrating social theory into public health practice. American Journal of Public Health,

Nova York, v. 95, n. 4, p. 591-595, 2005. 
SANTOS, I. S.; GOLDSTEIN, R. A.; RABELLO, A. Trajetória da Rede PDTSP-Teias: aprendizados e desafios de um modelo de gestão de pesquisa para soluções em saúde pública. In: SANTOS, I. S.; GOLDSTEIN, R. A. (Org.). Rede de Pesquisas em Manguinhos: sociedade, gestores e pesquisadores em conexão com o SUS. São Paulo: Hucitec, 2016. p. 27-54.

SCHWANDT, T. A. Três posturas epistemológicas para a investigação qualitativa: interpretativismo, hermenêutica e construcionismo social. In: DENZIN,

N. K.; LINCOLN, Y. S. (Org.). O planejamento da

pesquisa qualitativa: teorias e abordagens. 2. ed. Porto
Alegre: Artmed, 2006. p. 193-217.

THIOLLENT, M. Notas Para o Debate Sobre PesquisaAção. In: BRANDÃO, C. R. (Org.). Repensando a

Pesquisa Participante. 3. ed. São Paulo: Brasiliense, 1987. p. 82-103.

Recebido para publicação em abril de 2016

Versão final em setembro de 2016

Conflito de interesses: inexistente

Suporte financeiro: Fiotec - Projeto VPPLR Fio 13 